\title{
EFECTO DEL KETOPROFENO SOBRE LA PRESIÓN ARTERIAL PULMONAR EN TERNEROS JERSEY SOMETIDOS A HIPOXIA DE LA ALTURA
}

\author{
Effect of Ketoprofen on the Pulmonary Arterial Pressure in Jersey Calves \\ SubJected to High Altitud Hypoxia
}

\author{
Boris Lira M. ${ }^{1,3}$, Milder Ayón S. ${ }^{1}$, Sergio Cueva M. ${ }^{1}$, María Vásquez C. ${ }^{1}$, \\ Carlos Arana D. ${ }^{2}$ y Neiser Ocampo N. ${ }^{1}$
}

\section{Resumen}

Se estudió el efecto del ketoprofeno, un antiprostaglandínico bloqueador de la enzima ciclooxigenasa, sobre la presión arterial pulmonar media (PAPm) en 10 terneros Jersey machos, de 1 a 2 meses de edad, nacidos a nivel del mar y expuestos durante 3 días a una hipoxia ambiental de 3,320 m de altitud. Los terneros se distribuyeron en un grupo de 5 animales que recibió placebo (grupo control) y un grupo que recibió ketoprofeno en dosis de $3 \mathrm{mg} / \mathrm{kg}$ de peso vivo, por 5 días consecutivos, durante su estadía a nivel del mar (grupo tratado). La PAPm se determinó mediante la técnica de cateterismo cardiaco a nivel del mar y al tercer día de su estadía en la altura. Los valores promedio de la PAPm $(\mathrm{mm} \mathrm{Hg})$ a nivel del mar fueron de $19.00 \pm 0.94$ para el Grupo control y $21.00 \pm 3.39$ para el Grupo tratado, y en la altura de $39.50 \pm 6.00$ para el Grupo control y $31.25 \pm 5.70$ para el Grupo tratado, no existiendo diferencia significativa. Sin embargo, se observó diferencia por exposición a la altura al analizar la información de ambos grupos en conjunto (a nivel del mar: $20.00 \pm 2.68$ y al tercer día de exposición a la altura: $35.28 \pm 7.16)(\mathrm{p}<0.05)$. Estos resultados sugieren que el ketoprofeno no tuvo efecto sobre la PAPm en terneros Jersey sometidos durante 3 días a la hipoxia de la altura.

Palabras clave: ketoprofeno, terneros Jersey, presión arterial pulmonar media, cateterismo cardiaco, hipoxia

\section{Abstract}

The effect of ketoprofen, an antiprostaglandin substance that inhibits the enzyme ciclooxigenase, on the mean pulmonary arterial pressure (mPAP) was evaluated in 10, male Jersey calves, of 1-2 months of age. Calves were born at sea level and exposed during 3 days to an environmental hypoxia of 3,320 $\mathrm{m}$ of high altitude. A group of 5 animals received destiled water as placebo (Control group), and other group received ketoprofen in dose of $3 \mathrm{mg} / \mathrm{kg}$ body weight for 5 consecutive days while at sea level

${ }^{1}$ Laboratorio de Fisiología Animal, ${ }^{2}$ Estación Experimental del Centro de Investigaciones IVITA-El Mantaro, Facultad de Medicina Veterinaria, Universidad Nacional Mayor de San Marcos, Lima ${ }^{3}$ E-mail: borislira@yahoo.com 
(Treated group). The mPAP was measured by cardiac cateterism at sea level and 3 days after arriving to the high altitude. The mean values of mPAP $(\mathrm{mm} \mathrm{Hg})$ at sea level were: Control group $19.00 \pm 0.94$ and Treated group $21.00 \pm 3.39$; whereas at the high altitude were: Control group $39.50 \pm 6.00$ and Treated group $31.25 \pm 5.70$, and without significant differences. When data of the two groups were joined and analysed together, the values for mPAP at sea level $(20.00 \pm 2.68)$ were statistically different $(\mathrm{p}<0.05)$ from those at day 3 of exposition to high altitude $(35.28 \pm 7.16)$. The results suggested that ketoprofen had not effect on mPAP in Jersey calves exposed during 3 days at high altitude hypoxia.

Key words: ketoprofen, Jersey calves, mean pulmonary arterial pressure, cardiac cateterism, hypoxia

\section{INTRODUCCIÓN}

Las zonas de altura se caracterizan por la baja presión de oxígeno y el frío, los cuales afectan a la mayoría de las especies animales, siendo el bovino una de las más susceptibles. La respuesta más característica a estas condiciones es la vasoconstricción de la arteria pulmonar, que conlleva a una hipertensión arterial pulmonar (Ayón y Cueva, 1998). En los valles interandinos de la Sierra peruana se han introducido razas bovinas especializadas como la Holstein y el Brown Swiss para cruzarlas con el ganado criollo; sin embargo, la hipoxia y el frío les afectan desencadenando una hipertensión arterial pulmonar. El incremento de la resistencia vascular pulmonar produce hipertrofia y dilatación cardiaca e insuficiencia cardiaca congestiva derecha que son las características de la enfermedad de mal de altura (Cueva, 1968; Velásquez, 1988).

La hipertensión pulmonar observada se debe principalmente a la vasoconstricción de arteriolas pulmonares distales (Sylvester, 2001) y podría estar relacionado a la viscosidad sanguínea, por el aumento de la producción de eritropoyetina por las células intersticiales peritubulares renales, que tienen por función activar la masa de los glóbulos rojos y la concentración de hemoglobina (Ebert y Bunn, 1999). En casos de hipoxia prolongada se observa, además, remodelación estructural de las arterias pulmonares distales que aumentan aun más la resistencia vascular pulmonar (Brij y Peacock, 1998; Weissmann et al., 2001).
La hipoxia a nivel del endotelio vascular activa sustancias vasoconstrictoras como las prostaglandinas (PGs), y entre ellas a la PG E ${ }_{2}$, $\mathrm{PGD}_{2}$ y la $\mathrm{PGF}_{2 \propto}$, así como tromboxanos y la endotelina 1 (Earley et al., 2002; Yang et al., 2002).

Tanto las PGs y los tromboxanos derivan del ácido araquidónico por acción de la enzima ciclooxigenasa (COX), las que se presentan en 2 isoformas: la COX-1 que está presente en condiciones fisiológicas y la COX2 que se expresa como respuesta a citocinas proinflamatorias (González et al., 2002). El ketoprofeno es un antiinflamatorio no esteroideo que inhibe ambas isoformas de la COX y de esa manera bloquea su acción sobre el ácido araquidónico (González et al., 2002). Por tal motivo, se desarrolló el presente estudio con el objetivo de evaluar el efecto del ketoprofeno sobre la presión arterial pulmonar en animales que fueron sometidos a hipoxia de la altura.

\section{MATERIALeS y Métodos}

El presente estudio se realizó en dos localidades. La fase de altura se hizo en la Estación Experimental del Instituto Veterinario de Investigaciones Tropicales y de Altura (IVITA) - El Mantaro, a una altura de 3,320 msnm y con una presión barométrica de 510 mm $\mathrm{Hg}$ y una presión parcial de oxígeno de $107 \mathrm{~mm} \mathrm{Hg}$; y el estudio a nivel del mar fue en la Facultad de Medicina Veterinaria de la Universidad Nacional Mayor de San Mar- 
Cuadro 1. Efecto del ketoprofeno sobre la presión arterial pulmonar media (mm $\mathrm{Hg}$ ) a nivel del mar y al tercer día de exposición a la altura en terneros Jersey

\begin{tabular}{lccc}
\hline Grupos & $\begin{array}{c}\text { Animales } \\
(\mathrm{n})\end{array}$ & $\begin{array}{c}\text { A nivel del mar } \\
(\mathrm{x} \pm \mathrm{IC})^{1}\end{array}$ & $\begin{array}{c}\text { En altura } \\
(\mathrm{x} \pm \mathrm{IC})\end{array}$ \\
\hline Control & 5 & $19.00 \pm 0.94$ & $39.50 \pm 6.00$ \\
Tratado & 5 & $21.00 \pm 3.39$ & $31.25 \pm 5.70$ \\
\hline Total & 10 & $20.00 \pm 2.68$ & $35.28 \pm 7.16$ \\
\hline
\end{tabular}

${ }^{1}$ Promedio \pm intervalo de confianza del $95 \%$

${ }^{2}$ Al tercer día de exposición a la altura (3,320 msnm)

cos, a 160 msnm y con una presión barométrica de $750 \mathrm{~mm} \mathrm{Hg}$ y una presión parcial de oxígeno de $157 \mathrm{~mm} \mathrm{Hg}$.

Se utilizaron 10 terneros machos de la raza Jersey, de 1 a 2 meses de edad, nacidos a nivel del mar. Los animales se dividieron en dos grupos:

- Grupo 1: Animales control que recibieron placebo.

- Grupo 2: Animales tratados con ketoprofeno, a dosis de $3 \mathrm{mg} / \mathrm{kg}$ de peso vivo, vía intramuscular, por 5 días consecutivos.

Al término del tratamiento con ketoprofeno a nivel del mar, se midió la presión arterial pulmonar media (PAPm) a todos los animales mediante la técnica del cateterismo cardiaco. Al séptimo día de iniciado el tratamiento, los animales fueron transportados a la localidad de altura, considerándose este día como el primer día de exposición a la altura. Al tercer día de exposición de los animales a la altura se realizó otra medición de la PAPm.

La variable PAPm se analizó mediante la prueba estadística de análisis de varianza para evaluar si existe diferencia estadística por el efecto del tratamiento con ketoprofeno.

\section{Resultados}

No se encontró diferencia estadística significativa en PAPm entre los grupos en estudio por efecto del tratamiento con ketoprofeno a nivel del mar ni al tercer día de exposición a la altura; sin embargo, los valores absolutos de la PAP en la altura tuvieron una tendencia a la disminución en el grupo tratado (Cuadro 1).

El análisis estadístico tomando ambos grupos en conjunto mostró diferencia estadística significativa $(\mathrm{p}<0.05)$ por efecto de la altura. La PAPm se elevó en la altura en un $56.7 \%$ (Cuadro 1).

\section{Discusión}

El tratamiento por cinco días con ketoprofeno en terneros Jersey no influyó en la PAPm a nivel del mar ni al tercer día de exposición a la altura. El ketoprofeno es un antiinflamatorio no esteroideo, bloqueador inespecífico de la enzima ciclooxigenasa. Esta enzima es la responsable de catalizar los pasos comprometidos en el metabolismo del ácido araquidónico liberado en la respuesta inflamatoria a partir de los fosfolípidos de la membrana celular por la acción de la enzima 
Fosfolipasa A2 hasta la formación de prostaglandinas y tromboxanos (González et al., 2002; Ichinose et al., 2002).

La vasoconstricción pulmonar hipóxica es rápida. Inicialmente se debe a la liberación de sustancias vasoactivas como las prostaglandinas y tromboxanos. También se debe a los niveles per se de oxígeno y el estado redox, que llevan a la inhibición del flujo de potasio a través de la membrana celular, ocasionando la despolarización, que a su vez abren canales de calcio que conllevan al aumento de la concentración de calcio intracelular, ocasionando la contracción de la célula muscular lisa vascular (Peers, 1997; Dumas et al., 1999; Sylvester, 2001; Webb, 2003).

Los valores de PAPm obtenidos en la altura (Cuadro 1) estuvieron elevados y similares a los obtenidos por Tucker et al. (1975) y Will et al. (1975) en condiciones similares. Para Ames (2001), estos valores de PAPm están dentro del rango aceptable para introducir ganado bovino en la altura (PAPm $<41$ $\mathrm{mm} \mathrm{Hg}$ ) pudiendo considerarse como un buen indicador. Los valores de la PAPm en bovinos de la raza Holstein y Brown Swiss de 4 a 8 meses de edad encontrados por Ocampo (1997) fueron de 58.75 y $45.00 \mathrm{~mm} \mathrm{Hg}$, respectivamente, los cuales son mayores a los encontrados en el presente estudio en bovinos Jersey. Estos resultados sugieren una mejor respuesta adaptativa del bovino Jersey a cuadros hipóxicos, pero se debe considerar otros aspectos como procedencia, edad y susceptibilidad.

\section{Conclusiones}

- El tratamiento con ketoprofeno en los terneros Jersey no influyó significativamente $(p>0.05)$ en los valores de la PAPm a nivel del mar y a los tres días de exposición a la altura.
- La exposición de los terneros Jersey a la hipoxia de la altura por tres días incrementó $(\mathrm{p}<0.05)$ el valor promedio de la PAPm en un $56.7 \%$.

\section{Literatura Citada}

1. Ames DR. 2001. Guide for using pulmonary artery pressure (PAP) for beef cattle. Animal Sciences Research Report. p 1-3.

2. Ayón M, Cueva S. 1998. Adaptación del ganado bovino a la altura. Pub. Téc. $\mathrm{N}^{\mathrm{o}}$ 38. Facultad de Medicina Veterinaria, Univ. Nacional Mayor de San Marcos. Lima. 15 p.

3. Brij SO, Peacock AJ. 1998. Cellular responses to hypoxia in the pulmonary circulation. Thorax 53: 1075-1079.

4. Cueva S. 1968. Mal de altura en bovinos: Consideraciones generales, recomendaciones. $3^{\text {r }}$ Bol. Ext. UNMSMIVITA: 285-293.

5. Dumas JP, Bardou M, Goirand F, Dumas M. 1999. Hypoxic pulmonary vasoconstriction. Gnral Pharmacol 33: 289-297.

6. Earley S, Nelin LD, Chicoine LG, Walker BR. 2002. Hypoxia-induced pulmonary endothelin-1 expression is unaltered by nitric oxide. J Appl Physiol 92: 1152-1158.

7. Ebert BL, Bunn HF. 1999. Regulation of the erythropoietin gene. Blood 94: 1864-1877.

8. González, P.R.; G.P. Poza; R. Vives; G. Canto. 2002. Antiinflamatorios inhibidores selectivos de la cicloxigenasa2 (COX-2). Alergol. Inmunol. Clin. 17: 247-254.

9. Ichinose F, Ullrich R, Sapirstein A, Jones RC, Bonventre JV, Serhan CN, Bloch KD, Zapol WM. 2002. Cytosolic phospholipase A2 in hypoxic pulmonary vasoconstriction. J Clin Invest 109: 14931500. 
10. Ocampo J. 1997. Efecto del ketoprofeno sobre la presión arterial pulmonar en bovinos de altura. Tesis de Bachillerato. Lima: Facultad de Medicina Veterinaria, Univ. Nacional Mayor de San Marcos. 48 p.

11. Peers C. 1997. Oxygen-sensitive ion channels. Trends Pharmacol Sci 18: 405408.

12. Sylvester JT. 2001. Hypoxic pulmonary vasoconstriction. A radical view. Circ Res 88: 1228-1230.

13. Tucker A, McMurtry IF, Reeves JT, Alexander AF, Will DH, Grover RF. 1975. Lung vascular smooth muscle as a determinant of pulmonary hypertension at high altitude. Am J Physiol 228: 762767.

14. Velásquez L. 1988. Mal de montaña / sickness of high altitude. Gac Med Boliv 12: 70-73.
15. Webb RC. 2003. Smooth muscle contraction and relaxation. Adv Physiol Education 27: 201-206.

16. Weissmann $N$, Grimminger $F$, Olschewski A, Seeger W. 2001. Hypoxic pulmonary vasoconstriction: a multi-factorial response? Am J Physiol Lung Cell Mol Physiol 281: L314-L317.

17. Will DH, Hicks JL, Card CS, Alexander AF. 1975. Inherited susceptibility of cattle to high-altitude pulmonary hypertension. J Appl Physiol 38: 491-494.

18. Yang $X$, Sheares KKK, Davie N, Upton PD, Taylor GW, Horsley J, Wharton J, Morrell NW. 2002. Hypoxic induction of COX-2 regulates proliferation of human pulmonary artery smooth muscle cells. Am J Respir Cell Mol Biol 27: 688-696. 